\title{
Impact of surfaces on the optical properties of GaAs nanowires
}

\author{
O. Demichel, ${ }^{1,2, a)}$ M. Heiss, ${ }^{1,3}$ J. Bleuse, ${ }^{2}$ H. Mariette, ${ }^{2}$ and A. Fontcuberta i Morral ${ }^{1,3}$ \\ ${ }^{1}$ Laboratoire des Matériaux Semiconducteurs, Institut des Matériaux, École Polytechnique Fédérale de \\ Lausanne, 1015 Lausanne, Switzerland \\ ${ }^{2}$ CEA-CNRS Group, "Nanophysique et Semiconducteur," CEA/INAC/SP2M, 17 rue des Martyrs, \\ Fr-38054 Grenoble, France \\ ${ }^{3}$ Physik Department and Walter Schottky Institut, Technische Universitat Muenchen, Am Coulombwall 3, \\ 80578 Garching, Germany
}

(Received 15 October 2010; accepted 1 November 2010; published online 19 November 2010)

\begin{abstract}
The effect of surfaces on the optical properties of GaAs nanowires is evidenced by comparing nanowires with or without an AlGaAs capping shell as a function of the diameter. We find that the optical properties of unpassivated nanowires are governed by Fermi-level pinning, whereas, the optical properties of passivated nanowires are mainly governed by surface recombinations. Finally, we measure a surface recombination velocity of $3 \times 10^{3} \mathrm{~cm} \mathrm{~s}^{-1}$ one order of magnitude lower than values previously reported for $\{110\}$ GaAs surfaces. These results will serve as guidance for the application of nanowires in solar cell and light emitting devices. () 2010 American Institute of Physics. [doi:10.1063/1.3519980]
\end{abstract}

Semiconducting nanowires (NWs) are a topic of intense research as they offer the opportunity to explore properties of one-dimensional electronic systems. The understanding and the mastering of the electronic properties of such onedimensional systems are essential to achieve high efficiency devices. In particular, with the increase of the surface/volume ratio, the electronic properties of NW based devices become strongly dependent on the surface electronic states that can alter their electronic properties. Two main surface effects are reported in literature: surface states can act as recombination centers for free carriers ${ }^{1,2}$ or as surface charged traps. ${ }^{3,4}$ The first effect has recently been quantified by an original optical method for silicon NWs. ${ }^{5,6}$ The surface charge traps induce a pinning of the Fermi-level at the surface. A depletion shell appears and the electronic channel available for carriers is narrowed. Experimental proof of this effect in semiconducting NWs was observed by photoconductivity or capacitancevoltage measurements. ${ }^{7-10}$

In this letter, we present our results on the influence of surfaces on GaAs NWs measured by low temperature microphotoluminescence ( $\mu$-PL) spectroscopy. A systematic comparison is done between unpassivated NWs and those that were capped with a shell of $\mathrm{Al}_{0.4} \mathrm{Ga}_{0.6} \mathrm{As}$. Moreover, we take advantage of the tapered shape of the NWs to understand the role of diameter and surface/volume ratios in the luminescence efficiency. We demonstrate that capping directly modifies the recombination velocity and that passivated NW electronic properties are governed by surface recombinations, whereas, unpassivated NWs are strongly depleted by the Fermi-level pinning at the surface induced by charged surface trap states. This work will serve as a guidance for the passivation of the NW surfaces, which is of crucial importance for applications in laser and solar cell technology. ${ }^{11}$

GaAs NWs were synthesized by molecular beam epitaxy. The growth conditions are described elsewhere. ${ }^{12-14}$ The NWs have a prismatic shape and exhibit a pure zincblende GaAs crystal structure oriented along the (111) direc-

\footnotetext{
${ }^{a)}$ Author to whom correspondence should be addressed. Electronic mail: olivier.demichel@epfl.ch.
}

tion. Surfaces are $\{110\}$ oriented. ${ }^{15,16}$ The NWs are extremely long $(16 \mu \mathrm{m})$ and present a tapered shape (diameters go from $140 \mathrm{~nm}$ at the base to $70 \mathrm{~nm}$ at the tip). We investigated two samples which differ only by the presence or absence of a capping shell around the GaAs core. This shell is composed of a $4 \mathrm{~nm} \mathrm{Al}_{0.4} \mathrm{Ga}_{0.6} \mathrm{As}$ layer, which acts as an electronic barrier for the carriers generated in the NW core. This shell is surrounded by a $4 \mathrm{~nm} \mathrm{GaAs}$ layer to protect the $\mathrm{Al}_{0.4} \mathrm{Ga}_{0.6}$ As layer from oxidation. The optical properties of single, capped and uncapped NWs were explored along the NW by continuous-wave and time-resolved $\mu$-PL (TRPL) spectroscopy at liquid helium temperature. In the continuous-wave $\mu$-PL experiments, the NWs were excited via a semiconductor laser diode emitting at $780 \pm 10 \mathrm{~nm}$. The luminescence was detected and analyzed by the combination of a grating spectrometer and Si-charge coupled device. Mapping of PL was performed by moving the sample in an $\mathrm{x}-\mathrm{y}$ grid with a step size of $200 \mathrm{~nm}$ and acquiring the individual spectrum at each position. In TRPL experiments, single NWs were excited at $3.09 \mathrm{eV}$ with a frequencydoubled Ti-sapphire laser. The PL signal was detected by a standard streak camera setup. NWs were cooled down in contact with a cold finger. In both setups, samples were scanned below a confocal objective with piezopositioners. NW diameters were measured either by atomic force microscopy (AFM) or scanning electron microscopy (SEM).

Figure 1 compares the continuous-wave $\mu$-PL of capped and uncapped NWs. An AFM scan of a substrate region presenting 4 uncapped NWs is shown in Fig. 1(a). From the measured height, we extract the diameter along the NW axis. In Fig. 1(b), a mapping of the $\mu$-PL signal of the NWs pertaining to the same region is presented. One should note that the luminescence signal is mainly observed at the base of the NW and the PL line, corresponding to the radiative recombination of free excitons in pure zinc-blende GaAs crystal. An example of the integrated intensity of a capped NW is presented in Fig. 1(c). There, signal is detected along the whole NW length, though a decrease of intensity is observed from the NW base to the tip. Representative values of the intensity profiles along the NW axis for capped and un- 

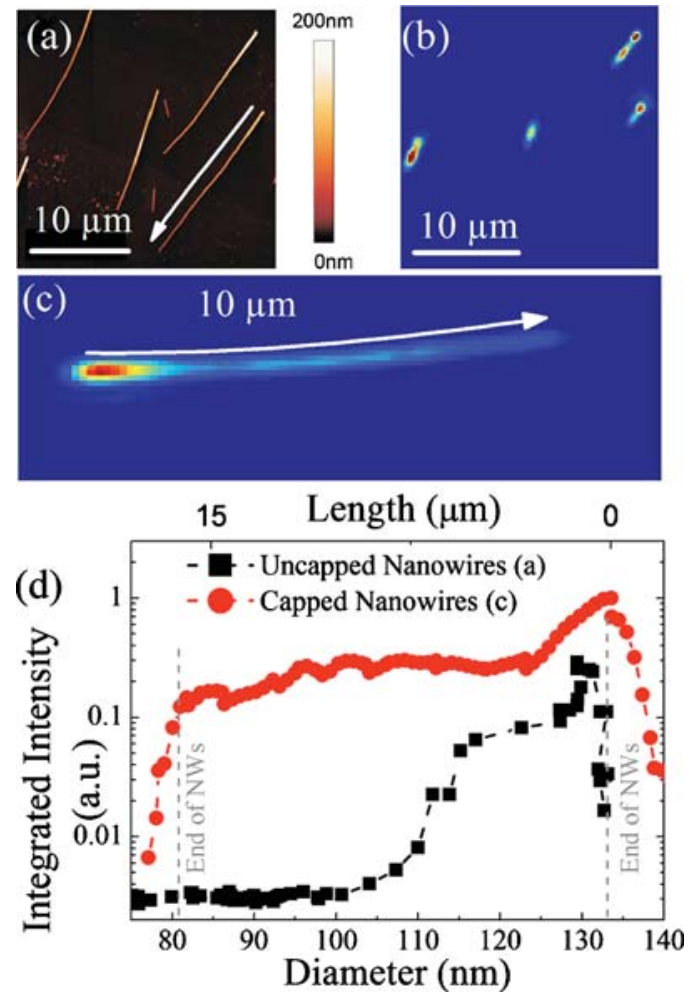

FIG. 1. (Color online) (a) AFM mapping of uncapped NWs transferred to a $\mathrm{SiO} 2$ substrate. (b) and (c) Mapping of integrated $4.2 \mathrm{~K} \mu$-PL signal of uncapped and capped NWs. The measurements (a) and (b) are performed on the same area of the sample. (d) Integrated PL signal along the length of capped (red circles) and uncapped NWs (black squares), the white arrows of (b) and (c) indicate the studied NWs and the direction of the $\mu$-PL scan. The curves are normalized to the maximum intensity of capped NWs.

capped NWs are plotted in Fig. 1(d). As the NWs are tapered, the curves also represent the diameter dependence of the PL integrated intensity. One can confirm that uncapped NWs exhibit luminescence only at the base while capped NWs are luminescent along their entire length. In particular, for uncapped NWs, PL signal is reduced to the noise level for diameters below a critical value of $\sim 100 \mathrm{~nm}$.

To further understand the influence of surfaces and interfaces on the optical properties of the NWs, we performed spatially resolved TRPL on the capped and uncapped NWs. Figure 2(b) shows an example of the dynamic response of the PL spectrum at the two extremities of a capped NW. The PL intensity decay time is clearly longer for the thickest extremity. The inverse lifetime as a function of the inverse radii is reported in Fig. 2(c) for three different capped NWs. According to Ref. 6, the mean slope of the gray dashed lines is linked to the surface recombination velocity (SRV) of the NWs. One should note that in the case of uncapped NWs, we measure SRV while the capped the recombination velocity corresponds to the GaAS/AlGaAs interface. To simplify the following, we will denote them in both cases of SRV. From this figure, we extract an SRV of $2.9 \times 10^{3} \pm 2$ $\times 10^{3} \mathrm{~cm} \mathrm{~s}^{-1}$ and a volume lifetime of $2.5 \pm 1.5 \mathrm{~ns}$ for capped NWs. This latter value is close to the typical carrier lifetime in bulk GaAs that demonstrates the high quality and purity of the GaAs crystal. Concerning the SRV, the value obtained here is one order of magnitude lower than the values reported for $\{110\}$ surfaces, ${ }^{17,18}$ and only one order of magnitude higher than values reported for $\{100\}$ GaAs surfaces passivated with an $\mathrm{Al}_{x} \mathrm{Ga}_{1-x}$ As layer: ${ }^{19}\{100\}$ surfaces

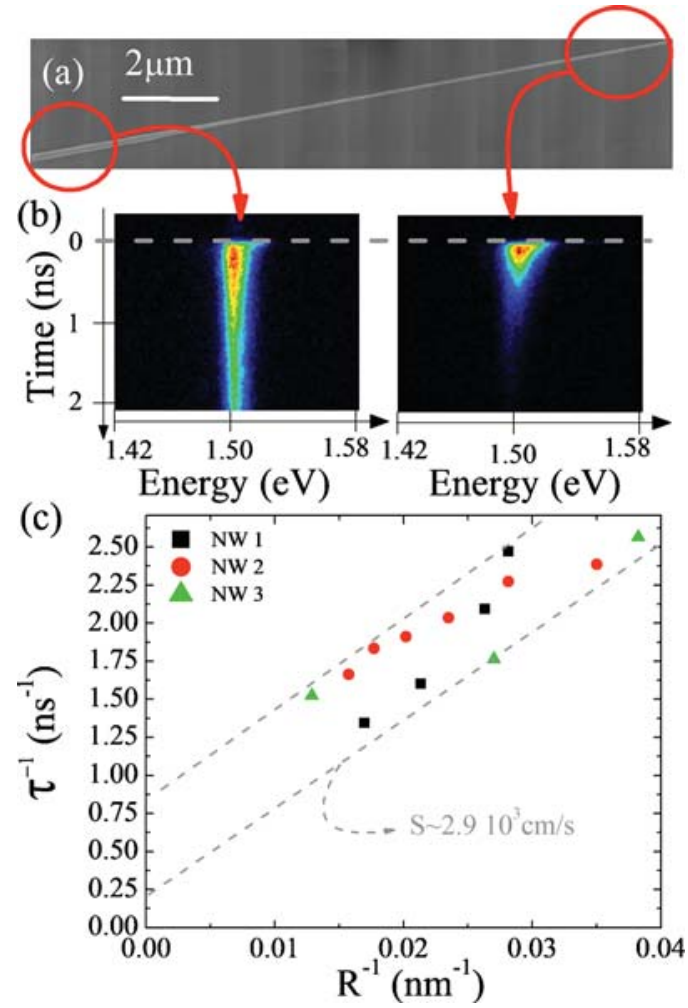

FIG. 2. (Color online) (a) SEM image of a single tapered capped NW. (b) TRPL spectra corresponding to the both extremities. For thinner radii, carrier lifetimes is shorter. (c) The inverse lifetime is reported as a function of the inverse radii for three different NWs.

are known to present lower densities of surface states and are easier to grow in high quality. It is important to note that the SRV reported here is state-of-the-art not only for the passivation of GaAs NWs, but also concerning the passivation of $\{110\} \mathrm{GaAs}$ surfaces. It corresponds to a density of surface recombination centers of $\sim 10^{12} \mathrm{~cm}^{-2}\left(\mathrm{~S} \sim v_{\text {th }} \sigma n_{\text {traps }}\right.$ where $v_{\text {th }}=4 \times 10^{6} \mathrm{~cm} \mathrm{~s}^{-1}$ is the thermal carrier velocity, $\sigma$ $\sim 10^{-15} \mathrm{~cm}^{2}$ is the typical surface trap cross section and $n_{\text {traps }}$ is the surface trap density). Uncapped NWs PL decay times are smaller than $30 \mathrm{ps,} \mathrm{which} \mathrm{would} \mathrm{correspond} \mathrm{to}$ SRVs higher than $10^{5} \mathrm{~cm} \mathrm{~s}^{-1}$ if electronic properties of uncapped NWs were governed by surface recombinations.

Now we turn to a more detailed discussion on the role of surface recombination in the diameter dependence of the PL intensity. Indeed, the existence of a critical diameter below which no PL signal is detected for uncapped NWs [Fig. 1(d)] cannot be explained by the existence of surface recombination even with SRV as high as $10^{5} \mathrm{~cm} \mathrm{~s}^{-1}$. Calarco et al. ${ }^{9}$ observed similar a behavior from photoconductivity experiments and explained this by the existence of surface charge traps that exchange charges with the semiconductor. Such traps can act as donors or acceptors depending on the Fermi level in the semiconductor. As a consequence, the Fermilevel is pinned ${ }^{20}$ and a depletion shell appears at the surface (as depicted in the inset of Fig. 3). At the limit where the radius of the NW is smaller than the depletion length, radiative recombinations are suppressed. For a clearer illustration of the role of surface depletion on the diameter dependent PL in capped and uncapped NWs, we plotted the PL intensity as a function of the diameter in a semilogarithmic plot shown in Fig. 3. The scattered points are experimental data from passivated and unpassivated NWs. The intensity is normalized 


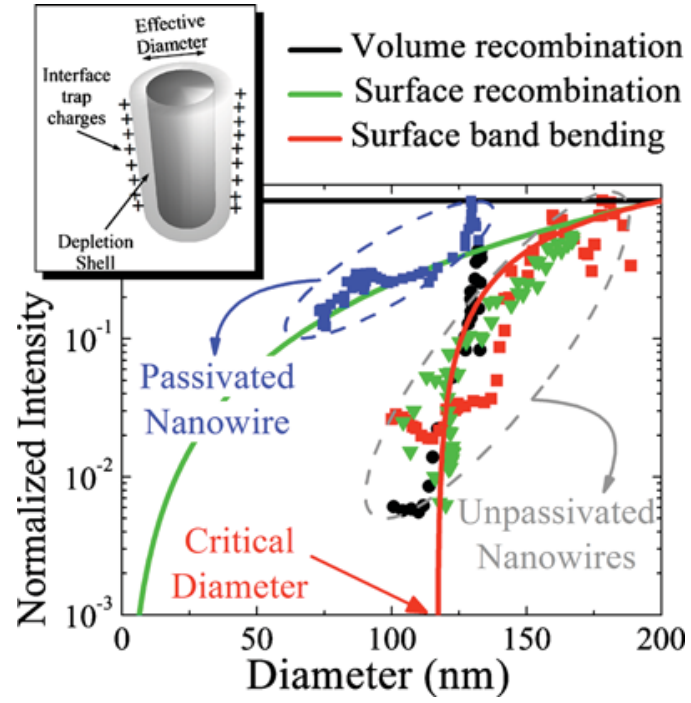

FIG. 3. (Color online) Comparison of the theoretical diameter dependences of PL intensity per unit volume (solid curves). The geometrical effect $\left(\propto r^{2}\right)$ resulting from a reduction in NW volume is already separated off. The curves correspond to the case of pure volume recombinations (black), pure surface recombinations (green) and to the case of surface band bending with volume recombinations (red). We added experimental data corresponding to passivated and unpassivated NWs. Data are normalized to the intensity of a NW diameter of $200 \mathrm{~nm}$. The inset is a schematic view of the depletion shell induced by a surface charge traps.

by a geometrical factor $\left(\sim r^{2}\right)$ that takes into account the volume reduction. The solid lines correspond to the theoretical diameter dependence of the PL intensity in three extreme cases: (i) absence of surface related effects (volume), (ii) prevalence of surface recombination and (iii) prevalence of surface band bending with a depletion thickness of $\sim 50 \mathrm{~nm}$.

The clustering of the experimental data for the uncapped NWs around the line showing the effect of the surface band bending clearly demonstrates that optical properties of uncapped NWs. These are well described by the Fermi-pinning at the surface that induces a depletion shell near the surface. The depletion shell is mostly affected by the charge trap surface density. In planar geometry, a depletion thickness of $50 \mathrm{~nm}$ corresponds [taking a barrier energy of the pinning of $0.5 \mathrm{eV}$ (Ref. 21)] to a charge trap surface density close to $\sim 10^{12} \mathrm{~cm}^{-2}$ that is in agreement with values reported for GaAs native oxidized surfaces. ${ }^{22}$

To conclude, we report $\mu$-PL experiments at a single NW level that compare luminescence intensity and the dynamics of uncapped and capped NWs. We have measured an SRV of $2.9 \times 10^{3} \mathrm{~cm} \mathrm{~s}^{-1}$ at the interface between the GaAs core and the $\mathrm{Al}_{0.4} \mathrm{Ga}_{0.6} \mathrm{As}$ shell that corresponds to the stateof-the-art for $\{110\}$ surfaces. We have also extracted a volume recombination carrier lifetime of $\sim 2.5 \mathrm{~ns}$ indicating the high crystal quality and purity of NWs. The luminescence in passivated and unpassivated NWs shows different diameter dependences. Unpassivated NWs present a surface charge trap density of $\sim 10^{12} \mathrm{~cm}^{-2}$ that induces depletion at the surface. No radiative recombination occurs below $100 \mathrm{~nm}$. On the contrary, carriers of passivated NWs mainly recombine through surface states with no depletion shell.

Authors thank G. Abstreiter and M. Bixhler for their experimental support. Funding from the ERC through the project 'UpCon', Marie Curie Excellence Grant SENFED and the DFG Nanosystems Initiative Munich are greatly acknowledged. Authors O. Demichel and M. Heiss contributed equally to this work.

${ }^{1}$ W. Shockley and W. T. Read, Phys. Rev. 87, 835 (1952).

${ }^{2}$ R. N. Hall, Phys. Rev. 87, 387 (1952).

${ }^{3}$ V. Schmidt, S. Senz, and U. Gösele, Appl. Phys. A: Mater. Sci. Process. 86, 187 (2007).

${ }^{4}$ V. Schmidt, J. V. Wittemann, S. Senz, and U. Gösele, Adv. Mater. (Weinheim, Ger.) 21, 2681 (2009).

${ }^{5}$ O. Demichel, V. Calvo, N. Pauc, A. Besson, P. Noé, F. Oehler, P. Gentile, and N. Magnea, Nano Lett. 9, 2575 (2009).

${ }^{6}$ O. Demichel, V. Calvo, A. Besson, P. Noé, B. Salem, N. Pauc, F. Oehler, P. Gentile, and N. Magnea, Nano Lett. 10, 2323 (2010).

${ }^{7}$ E. C. Garnett, Y.-C. Tseng, D. R. Khanal, J. Wu, J. Bokor, and P. Yang, Nat. Nanotechnol. 4, 311 (2009).

${ }^{8}$ K.-i. Seo, S. Sharma, A. A. Yasseri, D. R. Stewart, and T. I. Kamins, Electrochem. Solid-State Lett. 9, G69 (2006).

${ }^{9}$ R. Calarco, M. Marso, T. Richter, A. I. Aykanat, R. Meijers, A. v. d. Hart, T. Stoica, and H. Lüth, Nano Lett. 5, 981 (2005).

${ }^{10}$ S. Thunich, L. Prechtel, D. Spirkoska, G. Abstreiter, A. Fontcuberta i Morral, and A. W. Holleitner, Appl. Phys. Lett. 95, 083111 (2009).

${ }^{11}$ S. Kerboeuf, M. Bettiati, J. Gentner, C. Belouet, J. Perrière, J. Jimenez, and E. Martin, J. Electron. Mater. 28, 83 (1999).

${ }^{12}$ C. Colombo, D. Spirkoska, M. Frimmer, G. Abstreiter, and A. Fontcuberta i Morral, Phys. Rev. B 77, 155326 (2008).

${ }^{13}$ A. Fontcuberta i Morral, C. Colombo, G. Abstreiter, J. Arbiol, and J. R. Morante, Appl. Phys. Lett. 92, 063112 (2008).

${ }^{14}$ M. Heigoldt, J. Arbiol, D. Spirkoska, J. M. Rebled, S. Conesa-Boj, G. Abstreiter, F. Peiro, J. R. Morante, and A. Fontcuberta i Morral, J. Mater. Chem. 19, 840 (2009).

${ }^{15}$ D. Spirkoska, J. Arbiol, A. Gustafsson, S. Conesa-Boj, F. Glas, I. Zardo, M. Heigoldt, M. H. Gass, A. L. Bleloch, S. Estrade, M. Kaniber, J. Rossler, F. Peiro, J. R. Morante, G. Abstreiter, L. Samuelson, and A. Fontcuberta i Morral, Phys. Rev. B 80, 245325 (2009).

${ }^{16}$ I. Zardo, L. Yu, S. Conesa-Boj, S. Estradé, P. J. Alet, J. Rössler, and M. Frimmer, P. R. i Cabarrocas, F. Peirò, J. Arbiol, J. R. Morante, and A. F. i Morral, Nanotechnology 20, 155602 (2009).

${ }^{17}$ L. W. Tu, E. F. Schubert, M. Hong, and G. J. Zydzik, J. Appl. Phys. 80, 6448 (1996).

${ }^{18}$ E. A. Miller and G. L. Richmond, J. Phys. Chem. B 101, 2669 (1997).

${ }^{19}$ R. J. Nelson and R. G. Sobers, Appl. Phys. Lett. 32, 761 (1978).

${ }^{20}$ H. J. Stolz and G. Abstreiter, Solid State Commun. 36, 857 (1980).

${ }^{21}$ Y. Ishikawa, N. Tsurumi, T. Fukui, and H. Hasegawa, papers from the 25th Annual Conference on the Physics and Chemistry of Semiconductor Interfaces, 1998, Vol. 16, p. 2387.

${ }^{22}$ R. Fukasawa, M. Wakaki, K. Ohta, and H. Okumura, Jpn. J. Appl. Phys., Part 1 25, 652 (1986). 